\title{
不同恢复年限老参地土壤养分以及酚酸类代谢物 含量差异
}

李崇玮 ${ }^{1}$ 柏新富 ${ }^{1}$ 陈国忠 ${ }^{1}$ 朱 萍 $^{1}$ 张淑婷 $^{2}$ 侯玉平 $1^{*}$ 张兴晓 $^{1 *}$

鲁东大学生命科学学院, 山东烟台 $264025{ }^{2}$ 云南大学生态与环境学院, 昆明 650504

摘 要 人工种植西洋参(Panax quinquefolius)具有很高的经济效益, 但连作障碍已成为其产业可持续发展的限制因子。目前 对连作障碍成因的研究尚且不足。该研究以收获西洋参后恢复 $1 、 10 、 20$ 年的老参地(分别记为A $1 、 A 10 、 A 20)$ 为研究对象，以 未种植过西洋参的土地为对照 $(\mathrm{CK})$, 测定和分析土壤养分及酚酸类代谢物的变化, 以期从养分和化感作用的角度解析可能 造成西洋参连作障碍的关键因子。通过常规化学性质测定方法和气相色谱质谱联用(GC-MS)的方法测定土壤养分含量, 采用 高效液相色谱法(HPLC)测定土壤中的酚酸类代谢物含量。结果显示, 3组收获西洋参后的老参地的土壤pH均显著降低; A1 25 种有机态养分(氨基酸类、糖类和糖醇类物质)的含量显著降低, N-乙酰鸟氨酸、5-氨基戊酸、丝氨酸、亮氨酸、甘油和槐糖 等的含量均在所有老参地中显著下降, 经过 20 年轮作后依然不能恢复到对照水平。同时, 与预期相反, 被认为具有化感自毒 作用的酚酸类代谢物在收获西洋参后含量也显著下降, 其中, 香豆酸、原儿茶酸、阿魏酸和苯甲酸的含量在 $\mathrm{A} 1$ 中显著低于 $\mathrm{CK}$, 但经过 10 年时间轮作后可以恢复到接近对照水平。另外, $p$-香豆酸和丁香酸在A1、A10、A20的含量均显著低于 $\mathrm{CK}$, 即经过 20 年轮作依然不能恢复到对照水平; 酚酸类代谢物对西洋参生长的积极意义应被重视。相关性分析显示上述有机态养分含 量、 $\mathrm{pH}$ 和酚酸类代谢物含量之间大多数呈显著正相关关系, 表明各土壤特性之间存在密切的交互作用。综上所述, 种植西洋 参引起的土壤酸化、有机态养分和酚酸类代谢物含量降低及各因子间的协同作用可能是西洋参连作障碍的关键因素。

关键词 西洋参; 连作障碍; 有机态养分; 酚酸类代谢物; 高效液相色谱法(HPLC); 气相色谱质谱联用法(GC-MS)

李崇玮, 柏新富, 陈国忠, 朱萍, 张淑婷, 侯玉平, 张兴晓 (2021). 不同恢复年限老参地土壤养分以及酚酸类代谢物含量差异. 植物生态学报, 45 , 1263-1274. DOI: $10.17521 /$ cjpe.2020.0401

\section{Differences in soil nutrients and phenolic acid metabolites contents in American ginseng cul- tivated soils with different restoration years}

LI Chong-Wei ${ }^{1}$, BAI Xin-Fu ${ }^{1}$, CHEN Guo-Zhong ${ }^{1}$, ZHU Ping ${ }^{1}$, ZHANG Shu-Ting ${ }^{2}$, HOU Yu-Ping ${ }^{1 *}$, and ZHANG Xing-Xiao ${ }^{1 *}$

${ }^{1}$ College of Life Sciences, Ludong University, Yantai, Shandong 264025, China; and ${ }^{2}$ College of Ecology and Environment, Yunnan University, Kunming 650504, China

\section{Abstract}

Aims The cultivation of American ginseng (Panax quinquefolius) is of high economic benefits, but the continuous cropping has begun to limit the sustainable development of its industry. At present, the research on the problems of continuous cropping is limiting. In this study, the changes in soil nutrients and phenolic acid metabolites were measured and analyzed to investigate the key nutrient and allelopathic factors that may cause problems under continuous cropping of American ginseng, using the restoration plots for 1, 10, and 20 years after the long-term use of American ginseng cultivation (A1, A10, A20, respectively) and the control plot (CK) without cultivation of American ginseng.

Methods Soil nutrients contents were determined by conventional chemical methods and the gas chromatographymass spectrometry (GC-MS). Phenolic acid metabolites contents in soil were determined by the high performance liquid chromatography (HPLC).

Important findings The results showed that soil $\mathrm{pH}$ in the three groups of restoration plots was significantly

收稿日期Received: 2020-12-04 接受日期Accepted: 2021-07-05

基金项目: 山东省重大科技创新工程项目(2017GGH5129)、国家自然科学基金(31770581)、山东省高等学校科技计划项目(J17KA128)和山东省大学 生创新创业训练项目(201810451021)。Supported by the Major Science and Technology Innovation Project of Shandong Province (2017GGH5129), the National Natural Science Foundation of China (31770581), the Shandong Province Higher Educational Science and Technology Program (J17KA128), and Innovation and Entrepreneurship Training Project for college students in Shandong Province (201810451021).

* 通信作者Corresponding authors (Hou YP, hou_yuping@163.com; Zhang XX, zhangxingxiao@163.com) 
lower than the CK plots. The contents of 25 organic nutrients (amino acids, sugars and alcohols) in A1 were significantly decreased, and the contents of $\mathrm{N}$-acetylornithine, 5-aminovaleric acid, serine, leucine, glycerin and sophora in all restoration plots were significantly decreased compared to those in CK and had not returned to the control level even after 20 years of restoration. Simultaneously, contrary to expectations, the contents of coumaric acid, protocatechuic acid, ferulic acid and benzoic acid in A1 were significantly lower than those in CK, but could return to the control level after 10 years of restoration. In addition, the contents of $p$-coumaric acid and syringic acid in A1, A10 and A20 were significantly lower than those in CK, and even after 20 years of restoration, it had not returned to the control level. Attention should be paid to the positive effects of phenolic acid metabolites on the growth of American ginseng. The correlation analysis showed that most of the above organic nutrients contents, $\mathrm{pH}$ and phenolic acid metabolites contents showed significantly positive correlations among each other, indicating that there was a significant interaction between soil characteristics. In conclusion, soil acidification, decreases of organic nutrients contents and phenolic acids contents and synergistic effect of soil properties caused by cultivation of American ginseng may be the key factors leading to the problems of continuous cropping.

Key words Panax quinquefolius; continuous cropping obstacle; organic nutrients; phenolic acid metabolites; high performance liquid chromatography (HPLC); gas chromatography-mass spectrometry (GC-MS)

Li CW, Bai XF, Chen GZ, Zhu P, Zhang ST, Hou YP, Zhang XX (2021). Differences in soil nutrients and phenolic acid metabolites contents in American ginseng cultivated soils with different restoration years. Chinese Journal of Plant Ecology, 45, 1263-1274. DOI: $10.17521 /$ cjpe. 2020.0401

西洋参(Panax quinquefolius), 又名花旗参、洋 参、西洋人参, 是五加科人参属多年生宿根草本植 物, 原产于加拿大的魁北克和美国威斯康星州, 我 国20世纪70年代开始有计划地引进种植，因其具有 极高的药用价值(马宝兰等, 2011), 而广泛应用于食 品和药品领域, 人工种植面积不断扩大。然而, 西洋 参在种植过程中伴随着很强的连作障碍, 且其影响 可能长达数十年(简在友等, 2008), 给西洋参产业的 可持续发展造成极大的困扰(李丽和蒋景龙, 2018)。

连作障碍(忌地性)是指在同一块地(同一土壤) 连续种植同一种植物或近缘物种, 该植物会发生长 势变弱、产量与质量下降的现象(陈士林等, 2008)。 造成连作障碍的原因可能有: 土壤养分缺失(高微 微等, 2006b), 化感作用或自毒作用(张福锁和曹一 平, 1992; 高子勤和张淑香, 1998; He et al., 2009), 土壤微生物群落失调(Rahman \& Punja, 2005; Dong et al., 2017; Farh et al., 2018)。已有的关于连作障碍 的研究多集中于土壤微生物群落失调, 鉴定了多种 致病真菌, 如立枯丝核菌(Rhizoctonia solani)、茄病 镰刀菌(Fusarium solani)等(张国珍和张树峰, 2004; 毕武, 2008; Farh et al., 2018), 并且也发现了一些如 鞘氨醇单胞菌(Sphingomona) 等有益的土壤细菌。而 对于土壤养分缺失的研究多集中在无机养分和微量 元素上, 例如西洋参种植对氮 $(N)$ 、磷 $(P)$ 以及钾 $(K)$ 、 钲 $(\mathrm{Ca}) 、$ 锰 $(\mathrm{Mn})$ 等矿物营养元素的影响(孙贺, 2008; Li et al., 2020)等, 而同样为植物、菌根真菌以及细
菌提供养分的溶解性有机氮(Vitousek \& Matson, 1988; Fenn et al., 1998)、溶解性小分子糖类 (Momoshima et al., 1991; Kuzyakov \& Jones, 2006) 等土壤有机态养分的变化则鲜有研究。此外, 研究 者们对于土壤中化感物质变化的研究发现, 酚酸类 代谢物是造成连作障碍的重要因子, 高浓度的酚酸 类代谢物可以造成西洋参根系生物量的显著降低 (杨家学, 2009; Bi et al., 2010; 雷锋杰等, 2010; Jiao et al., 2012)。但是, 本课题组前期的研究发现, 部分 酚酸类代谢物在轮作 10 年的老参地土壤中的含量显 著降低(Li et al., 2021), 其他研究者的研究也有类 似结果(Jiao et al., 2012), 因此酚酸类物质在土壤中 的含量以及所起的作用仍需进一步研究。

同时，受连作障碍的困扰，收获后的老参地不 能继续种植西洋参, 而是进入一个轮作耕作模式以 改善土壤质量, 减少病虫害(张雪松, 2013)。然而, 目前对于收获西洋参后经过不同年限轮作土壤的恢 复情况, 特别是长时间序列的研究非常缺乏。为此, 本研究以收获西洋参后恢复 $1 、 10 、 20$ 年的老参地为 研究对象, 以未种植过西洋参的土地为对照 (CK), 测定和分析土壤无机和有机态养分及酚酸类代谢物 含量的变化, 试图解释不同恢复年限老参地土壤养 分发生的变化、酚酸类代谢物含量的变化以及各个 指标经过长期的轮作是否能恢复到未种植过西洋参 的对照水平。本研究通过对上述问题的回答来进一 步探究西洋参连作障碍的可能成因, 为西洋参连作 
障碍的解决提供一定的实验支持和理论指导。

\section{1 材料和方法}

\section{1 试验材料}

2019年4月进行土壤样品采集。采集地位于中国 西洋参主产区之一的山东省威海市文登区, 该区气 候属大陆性季风气候, 年平均气温 $11.9{ }^{\circ} \mathrm{C}$, 降水量 $816.2 \mathrm{~mm}$, 海拔 $45 \mathrm{~m}$ 。为了调查西洋参生长一茬 $($ 一 般种植3-5年)后以及收获西洋参后不同年限轮作的 老参地土壤性质的变化, 本研究选取4种样地类型: 没有种植过西洋参的农田(对照, CK), 收获西洋参 后1年(秋天收参, 第二年春天取样)的农田(A1), 收 获西洋参后轮作 10 年的农田(A10) 以及收获西洋参 后轮作 20 年的农田(A20)。每种类型样地设 10 个重复 (10块农田), 各采样地 (含收参后)均轮作小麦 (Triticum aestivum)、玉米(Zea mays)、花生(Arachis hypogaea) 等威海地区常见农作物, 人工管理相近,
其经纬度、海拔高度以及取样时地上作物和上茬作 物信息如表1所示。由于地块较大, 因此采用Z形多 点随机取样。在每一地块内选取靠里的位置(排除边 缘效应), 选择 10 个以上的采样点, 每个采样点取 0-20 cm的表层土壤约 $200 \mathrm{~g}$, 将各个取样点的土壤 混合作为一个样本。

\section{2 土壤化学性质与有机态养分的测定}

\subsection{1 土壤化学性质的测定}

土壤pH采用玻璃电极法测定, 土壤有机质含量 的测定采用高温外热重铬酸钾氧化-容量法, 土壤 全氮含量的测定采用开氏-蒸馏滴定法, 全磷含量 的测定采用氢氧化钠熔融-钼锑抗比色法, 碱解氮 含量的测定采用碱解扩散法，有效磷含量的测定采 用盐酸-氟化铵提取-钼锑抗比色法, 速效钾含量的 测定采用乙酸铵提取-火焰原子吸收分光光度法。以 上指标测定参考鲁如坤(2000)和鲍士旦(2000)的方 法。

表1 不同恢复年限样地的地理位置(经纬度与海拔高度)以及取样时地上作物和前茬作物

Table 1 Geographical location (longitude, latitude and altitude) of each plot, as well as current aboveground crops and the fore-rotating crops

\begin{tabular}{|c|c|c|c|c|}
\hline 序号 No. & $\mathrm{CK}$ & A1 & A 10 & A20 \\
\hline 1 & $\begin{array}{l}37.07^{\circ} \mathrm{N}, 122.12^{\circ} \mathrm{E}, 70 \mathrm{~m}, \\
\text { 小麦, 空地 } \\
\text { Triticum aestivum, Blank }\end{array}$ & $\begin{array}{l}37.07^{\circ} \mathrm{N}, 122.11^{\circ} \mathrm{E}, 70 \mathrm{~m} \\
\text { 小麦, 西洋参 } \\
\text { T. aestivum, Panax quinquefolius }\end{array}$ & $\begin{array}{l}37.07^{\circ} \mathrm{N}, 122.12^{\circ} \mathrm{E}, 50 \mathrm{~m} \\
\text { 小麦, 花生 } \\
\text { T. aestivum, Arachis hypogaea }\end{array}$ & $\begin{array}{l}37.07^{\circ} \mathrm{N}, 122.12^{\circ} \mathrm{E}, 40 \mathrm{~m} \\
\text { 空地, 花生 } \\
\text { Blank, A. hypogaea }\end{array}$ \\
\hline 2 & $\begin{array}{l}37.18^{\circ} \mathrm{N}, 122.24^{\circ} \mathrm{E}, 70 \mathrm{~m} \text {, } \\
\text { 空地, 空地 } \\
\text { Blank, Blank }\end{array}$ & $\begin{array}{l}37.07^{\circ} \mathrm{N}, 122.12^{\circ} \mathrm{E}, 70 \mathrm{~m}, \\
\text { 小麦, 西洋参 } \\
\text { T. aestivum, P. quinquefolius }\end{array}$ & $\begin{array}{l}37.07^{\circ} \mathrm{N}, 122.12^{\circ} \mathrm{E}, 50 \mathrm{~m} \\
\text { 空地, 玉米 } \\
\text { Blank, Zea mays }\end{array}$ & $\begin{array}{l}37.07^{\circ} \mathrm{N}, 122.12^{\circ} \mathrm{E}, 50 \mathrm{~m} \\
\text { 空地, 番薯 } \\
\text { Blank, Ipomoea batatas }\end{array}$ \\
\hline 3 & $\begin{array}{l}37.18^{\circ} \mathrm{N}, 122.23^{\circ} \mathrm{E}, 40 \mathrm{~m} \\
\text { 空地, 玉米 } \\
\text { Blank, Zea mays }\end{array}$ & $\begin{array}{l}37.07^{\circ} \mathrm{N}, 122.12^{\circ} \mathrm{E}, 70 \mathrm{~m} \\
\text { 小麦, 西洋参 } \\
\text { T. aestivum, P. quinquefolius }\end{array}$ & $\begin{array}{l}37.07^{\circ} \mathrm{N}, 122.12^{\circ} \mathrm{E}, 50 \mathrm{~m} \\
\text { 小麦, 花生 } \\
\text { T. aestivum, A. hypogaea }\end{array}$ & $\begin{array}{l}37.07^{\circ} \mathrm{N}, 122.12^{\circ} \mathrm{E}, 40 \mathrm{~m} \\
\text { 空地, 花生 } \\
\text { Blank, A. hypogaea }\end{array}$ \\
\hline 4 & $\begin{array}{l}37.18^{\circ} \mathrm{N}, 122.23^{\circ} \mathrm{E}, 40 \mathrm{~m} \\
\text { 空地, 花生 } \\
\text { Blank, Arachis hypogaea }\end{array}$ & $\begin{array}{l}37.07^{\circ} \mathrm{N}, 122.12^{\circ} \mathrm{E}, 70 \mathrm{~m} \\
\text { 小麦, 西洋参 } \\
\text { T. aestivum, P. quinquefolius }\end{array}$ & $\begin{array}{l}37.07^{\circ} \mathrm{N}, 122.12^{\circ} \mathrm{E}, 60 \mathrm{~m} \\
\text { 空地, 玉米 } \\
\text { Blank, Z. mays }\end{array}$ & $\begin{array}{l}37.07^{\circ} \mathrm{N}, 122.12^{\circ} \mathrm{E}, 50 \mathrm{~m} \\
\text { 空地, 花生 } \\
\text { Blank, A. hypogaea }\end{array}$ \\
\hline 5 & $\begin{array}{l}37.18^{\circ} \mathrm{N}, 122.23^{\circ} \mathrm{E}, 40 \mathrm{~m} \\
\text { 玉米, 小麦 } \\
\text { Z. mays, T. aestivum }\end{array}$ & $\begin{array}{l}37.18^{\circ} \mathrm{N}, 122.23^{\circ} \mathrm{E}, 40 \mathrm{~m} \\
\text { 小麦, 西洋参 } \\
\text { T. aestivum, P. quinquefolius }\end{array}$ & $\begin{array}{l}37.07^{\circ} \mathrm{N}, 122.12^{\circ} \mathrm{E}, 60 \mathrm{~m} \\
\text { 空地, 玉米 } \\
\text { Blank, Z. mays }\end{array}$ & $\begin{array}{l}37.07^{\circ} \mathrm{N}, 122.12^{\circ} \mathrm{E}, 50 \mathrm{~m} \\
\text { 空地, 花生 } \\
\text { Blank, A. hypogaea }\end{array}$ \\
\hline 6 & $\begin{array}{l}37.18^{\circ} \mathrm{N}, 122.23^{\circ} \mathrm{E}, 40 \mathrm{~m} \\
\text { 空地, 空地 } \\
\text { Blank, Blank }\end{array}$ & $\begin{array}{l}37.18^{\circ} \mathrm{N}, 122.23^{\circ} \mathrm{E}, 40 \mathrm{~m} \\
\text { 小麦, 西洋参 } \\
\text { T. aestivum, P. quinquefolius }\end{array}$ & $\begin{array}{l}37.07^{\circ} \mathrm{N}, 122.11^{\circ} \mathrm{E}, 70 \mathrm{~m} \\
\text { 空地, 玉米 } \\
\text { Blank, Z. mays }\end{array}$ & $\begin{array}{l}37.11^{\circ} \mathrm{N}, 122.07^{\circ} \mathrm{E}, 70 \mathrm{~m} \\
\text { 空地, 空地 } \\
\text { Blank, Blank }\end{array}$ \\
\hline 7 & $\begin{array}{l}37.19^{\circ} \mathrm{N}, 122.25^{\circ} \mathrm{E}, 40 \mathrm{~m} \\
\text { 小麦, 花生 } \\
\text { T. aestivum, A. hypogaea }\end{array}$ & $\begin{array}{l}37.18^{\circ} \mathrm{N}, 122.23^{\circ} \mathrm{E}, 40 \mathrm{~m} \\
\text { 小麦, 西洋参 } \\
\text { T. aestivum, P. quinquefolius }\end{array}$ & $\begin{array}{l}37.07^{\circ} \mathrm{N}, 122.11^{\circ} \mathrm{E}, 70 \mathrm{~m} \\
\text { 空地, 玉米 } \\
\text { Blank, Z. mays }\end{array}$ & $\begin{array}{l}37.11^{\circ} \mathrm{N}, 122.07^{\circ} \mathrm{E}, 70 \mathrm{~m} \\
\text { 空地, 玉米 } \\
\text { Blank, Z mays }\end{array}$ \\
\hline 8 & $\begin{array}{l}37.19^{\circ} \mathrm{N}, 122.25^{\circ} \mathrm{E}, 40 \mathrm{~m} \\
\text { 空地, 玉米 } \\
\text { Blank, Z. mays }\end{array}$ & $\begin{array}{l}37.18^{\circ} \mathrm{N}, 122.23^{\circ} \mathrm{E}, 40 \mathrm{~m} \\
\text { 小麦, 西洋参 } \\
\text { T. aestivum, P. quinquefolius }\end{array}$ & $\begin{array}{l}37.07^{\circ} \mathrm{N}, 122.12^{\circ} \mathrm{E}, 70 \mathrm{~m} \\
\text { 空地, 玉米 } \\
\text { Blank, Z. mays }\end{array}$ & $\begin{array}{l}37.11^{\circ} \mathrm{N}, 122.07^{\circ} \mathrm{E}, 0 \mathrm{~m} \\
\text { 空地, 花生 } \\
\text { Blank, A. hypogaea }\end{array}$ \\
\hline 9 & $\begin{array}{l}37.19^{\circ} \mathrm{N}, 122.25^{\circ} \mathrm{E}, 35 \mathrm{~m} \\
\text { 杂草, 玉米 } \\
\text { Weeds, Z. mays }\end{array}$ & $\begin{array}{l}37.18^{\circ} \mathrm{N}, 122.23^{\circ} \mathrm{E}, 40 \mathrm{~m} \\
\text { 小麦, 西洋参 } \\
\text { T. aestivum, P. quinquefolius }\end{array}$ & $\begin{array}{l}37.07^{\circ} \mathrm{N}, 122.12^{\circ} \mathrm{E}, 70 \mathrm{~m} \\
\text { 空地, 玉米 } \\
\text { Blank, Z. mays }\end{array}$ & $\begin{array}{l}37.13^{\circ} \mathrm{N}, 122.06^{\circ} \mathrm{E}, 60 \mathrm{~m} \\
\text { 空地, 玉米 } \\
\text { Blank, Z. mays }\end{array}$ \\
\hline 10 & $\begin{array}{l}37.19^{\circ} \mathrm{N}, 122.25^{\circ} \mathrm{E}, 35 \mathrm{~m} \\
\text { 杂草, 花生 } \\
\text { Weeds, A. hypogaea }\end{array}$ & $\begin{array}{l}37.18^{\circ} \mathrm{N}, 122.23^{\circ} \mathrm{E}, 40 \mathrm{~m} \\
\text { 小麦, 西洋参 } \\
\text { T. aestivum, P. quinquefolius }\end{array}$ & $\begin{array}{l}37.07^{\circ} \mathrm{N}, 122.12^{\circ} \mathrm{E}, 70 \mathrm{~m} \\
\text { 小麦, 花生 } \\
\text { T. aestivum, A. hypogaea }\end{array}$ & $\begin{array}{l}37.13^{\circ} \mathrm{N}, 122.06^{\circ} \mathrm{E}, 60 \mathrm{~m} \\
\text { 空地, 空地 } \\
\text { Blank, Blank }\end{array}$ \\
\hline
\end{tabular}

$\mathrm{A} 1$, 收获西洋参后轮作 1 年的土壤; $\mathrm{A} 10$, 收获西洋参后轮作 10 年的土壤; $\mathrm{A} 20$, 收获西洋参后轮作 20 年的土壤; CK, 未种植过西洋参的土壤。表中数据 顺序为纬度、经度、海拔、取样时地上作物以及前一茬作物。空地表示该地块无作物生长。

A1, 1-year post-ginseng rotation soil; A10, 10-year post-ginseng rotation soil; A20, 20-year post-ginseng rotation soil; CK, no ginseng cultivation history. The data is in the order of: latitude, longitude, altitude, aboveground crops at sampling time and fore-rotating crop. Blank means that there is no crop in the plot. 


\subsection{2 土壤有机态养分含量的测定}

土壤中易被植物直接吸收利用的氨基酸类、糖 类和糖醇类等小分子有机态养分含量的测定, 采用 气相色谱质谱联用法(GC-MS), 试剂以及具体步骤 如下。

1.2.2.1 有机态养分的提取 称取 $1 \mathrm{~g}$ 样本, 加入 $20 \mu \mathrm{L}$ 内标物(L-2-氯-苯丙氨酸, $0.3 \mathrm{mg} \cdot \mathrm{mL}^{-1}$, 甲醇 配置)和 $1 \mathrm{~mL}$ 甲醇水混合液(甲醇:水体积比 $1: 1$ ); 依 次加入两颗小钢珠, 在 $-20^{\circ} \mathrm{C}$ 放置 $2 \min$ 预冷, 加入 研磨机研磨 $(60 \mathrm{~Hz}, 2 \mathrm{~min})$; 将匀浆后的样品转移至 $15 \mathrm{~mL}$ 离心管中, 用 $1 \mathrm{~mL}$ 混合液(甲醇:水体积比 $1: 1$ ) 转移管壁残留, 重复3次(共 $3 \mathrm{~mL}$ ); 离心 $10 \mathrm{~min}$ $\left(7700 \mathrm{r} \cdot \mathrm{min}^{-1}, 4{ }^{\circ} \mathrm{C}\right)$ 取上清 $2.5 \mathrm{~mL} 5 \mathrm{~mL}$ 离心管中, 冻干; 用 $400 \mu \mathrm{L}$ 混合液(甲醇:水体积比 $1: 1$ )复溶, 浴 旋振荡 $60 \mathrm{~s}$, 超声 $30 \mathrm{~s}$, 将溶液转移至 $1.5 \mathrm{~mL}$ 离心管 中; 离心 $10 \mathrm{~min}\left(12000 \mathrm{r} \cdot \mathrm{min}^{-1}, 4{ }^{\circ} \mathrm{C}\right)$, 取 $300 \mu \mathrm{L}$ 的 上清液装入玻璃衍生瓶中; 质控样本 $(\mathrm{QC})$ 由所有样 本的提取液等体积混合制备而成, 每个 $\mathrm{QC}$ 的体积 与样本相同; 用冷冻浓缩离心干燥器冻干样品。向 玻璃衍生小瓶中加入 $80 \mu \mathrm{L}$ 的甲氧胺盐酸盐吡啶溶 液 $\left(15 \mathrm{mg} \cdot \mathrm{mL}^{-1}\right)$, 浴旋振荡 $2 \mathrm{~min}$ 后, 于 $37{ }^{\circ} \mathrm{C}$ 振荡培 养箱中进行肟化反应 $90 \mathrm{~min}$ 。将样品取出后再加入 $80 \mu \mathrm{L}$ 的BSTFA (含1\% TMCS) 衍生试剂和 $20 \mu \mathrm{L}$ 的正 己烷, 加入 11 种内标脂肪酸甲酯 $(\mathrm{C} 8 / \mathrm{C} 9 / \mathrm{C} 10 / \mathrm{C} 12$ / $\mathrm{C} 14 / \mathrm{C} 16,0.8 \mathrm{mg} \cdot \mathrm{mL}^{-1}$; C18/C20/ C22/C24/C26 (下 文备注), $0.4 \mathrm{mg} \cdot \mathrm{mL}^{-1}$, 均为氯仿配置) $10 \mu \mathrm{L}$, 浴旋 振荡 $2 \mathrm{~min}$ 后, 于70 ${ }^{\circ} \mathrm{C}$ 反应 $60 \mathrm{~min}$ 。取出样本后, 在室温放置30 min, 进行GC-MS分析。

备注: BSTFA为双 (三甲基硅烷基)三氟乙酰胺, TMCS 为三甲基氯硅烷; 11种脂肪酸甲酯分别为: 辛 酸甲酯(methyl octanoate, C8:0)、壬酸甲酯(methyl nonoate, C9:0)、癸酸甲酯(methyl caprate, C10:0)、十 二烷酸甲酯(methyldodecanoate, C12:0)、十四烷酸甲 酯(methyl myristate, C14:0)、十六烷酸甲酯(methyl hexadecanoate, C16:0)、十八烷酸甲酯(methylstearate, C18:0)、二十烷酸甲酯(methyl eicosanoate, C20:0)、 二十二烷酸甲酯(methyl behenate, C22:0)、二十四烷 酸甲酯(methyltetracosanoate, C24:0)、二十六烷酸甲 酯(methyl eicosanoate, C26:0)。

1.2.2.2 气相色谱-质谱分析条件 气相色谱条件: DB-5MS毛细管柱 $(30 \mathrm{~m} \times 0.25 \mathrm{~mm} \times 0.25 \mu \mathrm{m}$, Agilent J\&W Scientific, Folsom, USA), 载气为高纯
氮气(纯度不小于 $99.999 \%$ ), 流速 $1.0 \mathrm{~mL} \cdot \mathrm{min}^{-1}$, 进 样口的温度为 $260{ }^{\circ} \mathrm{C}$ 。进样量 $1 \mu \mathrm{L}$, 不分流进样, 溶 剂延迟 $5 \mathrm{~min}$ 。程序升温: 柱温箱的初始温度为 $60{ }^{\circ} \mathrm{C}$, 以 $8{ }^{\circ} \mathrm{C} \cdot \mathrm{min}^{-1}$ 程序升温至 $125{ }^{\circ} \mathrm{C}, 5{ }^{\circ} \mathrm{C} \cdot \mathrm{min}^{-1}$ 升温至 $210{ }^{\circ} \mathrm{C} ; 10{ }^{\circ} \mathrm{C} \cdot \mathrm{min}^{-1}$ 升温至 $270{ }^{\circ} \mathrm{C}, 20{ }^{\circ} \mathrm{C} \cdot \mathrm{min}^{-1}$ 升 温至 $305{ }^{\circ} \mathrm{C}$ 保持 $5 \mathrm{~min}$ 。

质谱条件: 电子轰击离子源(EI), 离子源温度 $230{ }^{\circ} \mathrm{C}$, 四级杆温度 $150{ }^{\circ} \mathrm{C}$, 电子能量 $70 \mathrm{eV}$ 。扫描 方式为全扫描模式(SCAN), 质量扫描范围: $\mathrm{m} / \mathrm{z}$ $50-500$ 。

\section{3 土壤酚酸类代谢物含量的测定}

\subsection{1 土壤酚酸类代谢物含量的提取}

土壤酚酸类代谢物的提取参照 Hartley 和 Buchan (1979)的方法, 稍加改良。具体步骤如下: 称 取 $30 \mathrm{~g}$ 过 40 目(孔径 $0.425 \mathrm{~mm}$ )筛的土样于雉形瓶中, 加入 $50 \mathrm{~mL} 1 \mathrm{~mol} \cdot \mathrm{L}^{-1} \mathrm{NaOH}$ 溶液, 振荡过夜 $(10 \mathrm{~h})$, 将雉形瓶内容物全部倒入离心管中, 离心 (3 $800 \mathrm{r} \cdot \mathrm{min}^{-1}$ ) $5 \mathrm{~min}$, 上清液用 $12 \mathrm{~mol} \cdot \mathrm{L}^{-1} \mathrm{HCl}$ 调整 $\mathrm{pH}$ 到2.0 (去除胡敏酸), 再次离心 $15 \mathrm{~min}$ (同上), 上 清液用乙酸乙酯萃取 3 次 $($ 每次 $20 \mathrm{~mL})$, 将上层乙酸 乙酯溶液加入圆底烧瓶内进行旋蒸至干燥。随后, 向圆底烧瓶加入 $1 \mathrm{~mL}$ 甲醇, 摇晃旋转至充分溶解后 过 $0.22 \mu \mathrm{m}$ 注射器滤膜, 滤液装入进样瓶以备上机 测定。

\subsection{2 标准溶液的配置和标准曲线的制作}

单个酚酸类代谢物标准溶液的配置: 准确称量 $10 \mathrm{mg}$ 没食子酸、香豆酸、原儿茶酸、对羟基苯甲 酸、绿原酸、香草酸、咖啡酸、丁香酸、香草醛、 对香豆酸、阿魏酸、苯甲酸、水杨酸、肉桂酸(雷锋 杰等, 2010; 焦晓林等, 2015b; 李自博等, 2016), 分 别置入 14 个 $100 \mathrm{~mL}$ 容量瓶中, 用甲醇溶解并定容至 $100 \mathrm{~mL}$, 则每种酚酸类代谢物标准溶液的浓度为 $100 \mu \mathrm{g} \cdot \mathrm{mL}^{-1}$ 。酚酸类代谢物标准品购自Sigma公司。

混合酚酸类代谢物标准溶液的配置: 准确称量 $10 \mathrm{mg}$ 没食子酸、香豆酸、原儿茶酸、对羟基苯甲 酸、绿原酸、香草酸、咖啡酸、丁香酸、香草醛、 对香豆酸、阿魏酸、苯甲酸、水杨酸、肉桂酸置入 $100 \mathrm{~mL}$ 容量瓶中, 用甲醇溶解混匀并定容至 $100 \mathrm{~mL}$, 则该标准溶液每种酚酸类代谢物的浓度为 $100 \mu \mathrm{g} \cdot \mathrm{mL}^{-1}$ 。用此溶液配置稀释 $2 、 5 、 10 、 20 、 50$ 倍的标准品溶液, 其浓度分别为 $50 、 20 、 10 、 5 、$ $2 \mu \mathrm{g} \cdot \mathrm{mL}^{-1}$, 配置完成后, 储存在 $4{ }^{\circ} \mathrm{C}$ 冰箱中待用。

www.plant-ecology.com 
Waters高效液相色谱系统(E2695, Waters, Milford, USA)中使用二极管阵列检测器, 使用Uranus $\mathrm{C} 18$ 柱 $(250 \mathrm{~mm} \times 4.6 \mathrm{~mm} \times 5 \mu \mathrm{m})$ 和保护柱 $(20 \mathrm{~mm} \times$ $4.6 \mathrm{~mm} \times 5 \mu \mathrm{m}$ ) 在 $280 \mathrm{~nm}$ 下进行检测分析。使用以 下流动相溶液进行高效液相色谱分离: 三氟乙酸水 溶液 (体积分数 $0.1 \%$ ) 和乙腈的流动相, 柱温 $30{ }^{\circ} \mathrm{C}$, 注射体积 $10 \mu \mathrm{L}$, 流速 $1.0 \mathrm{~mL} \cdot \mathrm{min}^{-1}$, 洗脱梯度是 5\%-10\%乙腈(0-10 min), 10\%-10\%乙腈(10-20 min), $10 \%-35 \%$ 乙 腈 $(20-60 \mathrm{~min}), 35 \%-60 \%$ 乙 腈 (60-75 min), 60\%-60\%乙腈(75-85 min), 60\%-5\%乙 腈 (85-86 $\mathrm{min}), 5 \%-5 \%$ 乙腈 $(86-90 \mathrm{~min}$ ) 并保持 $5 \mathrm{~min}$ 。每次进样间隔用流动相使柱平衡 $10 \mathrm{~min}$ 。将 配置好的不同浓度的标准品混合样品以及待测滤液 放入高效液相色谱系统, 使用Waters Empower工作 站(Waters, Milford, USA)记录和处理色谱数据, 以 不同酚酸类代谢物的保留时间为标准进行鉴别。得 到标准品峰的保留时间和峰面积(图1), 以峰面积为 因变量和进样标准品质量为自变量来制作标准曲线 方程。结果计算: 将待测滤液所测峰面积 $y$ 带入标准 曲线方程, 求得 $x$, 然后将 $x$ 代入下面的公式计算风 干土中所含酚酸类代谢物的浓度。

$$
a=\left(\frac{x}{10 \mu \mathrm{L}} \times 1000 \mu \mathrm{L}\right) \div 30
$$

式中, $a$ 为 $1 \mathrm{~g}$ 风干土中所含酚酸类代谢物的浓度 $\left(\mu \mathrm{g} \cdot \mathrm{g}^{-1}\right), x$ 为 $10 \mu \mathrm{L}$ 进样体积中所包含的某种酚酸的 质量 $(\mu \mathrm{g})$ 。

\section{4 数据分析}

采用统计软件SPSS 19.0中单因素方差分析和 Duncan多重比较检验不同样品各个指标间的差异 显著性; 同时, 利用R 4.0.2对不同组土壤所测的 44

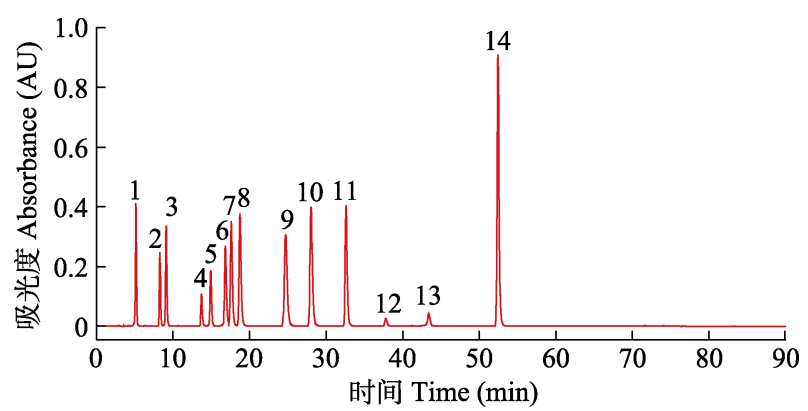

图1 高效液相色谱法得到的酚酸标样的出峰时间和出峰面 积。1, 没食子酸; 2 , 香豆酸; 3 , 原儿茶酸; 4 , 对羟基苯甲酸; 5 , 绿原酸; 6 , 香草酸; 7 , 咖啡酸; 8 , 丁香酸; 9 , 香草醛; 10 , 对香豆酸; 11, 阿魏酸; 12 , 苯甲酸; 13 , 水杨酸; 14 , 肉桂酸。 Fig. 1 Peak time and peak area of standard phenolicacids obtained by high performance liquid chromatography (HPLC). 1, gallic acid; 2, coumaric acid; 3, protocatechuic acid; 4, p-hydroxybenzonic acid; 5, chlorogenic acid; 6 , vanillic acid; 7, caffeic acid; 8, syringic acid; 9, vanillin (vanillic aldehyd); 10, p-coumaric acid; 11, ferulic acid; 12, benzoic acid; 13, salicylic acid; 14, cinnamic acid.

种氨基酸和糖醇类养分进行主成分分析(PCA)。采 用Excel 2016、Graphpad Prism 8、Adobe Illustrator CS6、Photoshop CS6等相关软件进行图片、图表处理。

\section{2 结果}

\section{1 不同恢复年限老参地土壤化学性质和有机态 养分含量的变化}

\subsection{1 土壤化学性质的变化}

与对照相比, 3组收获西洋参后的老参地土壤 $\mathrm{pH}$ 均显著下降 $(p<0.05)$; 收参后 1 年的老参地中有 效钾含量显著高于对照和轮作 10 年、 20 年的老参地 $(p<0.05)$, 而后三者之间差异不显著, 表明刚收获 西洋参后土壤有效钾含量较高, 经过 10 年轮作即可 回归到对照水平。土壤全氮、全磷、碱解氮、有效 磷的含量在各年限土壤间没有显著差异(表2)。

表2 不同恢复年限老参地土壤化学性质的比较(平均值土标准误, $n=10$ )

Table 2 Comparison of soil chemical properties in soils with different restoration years from the long-term cultivation of American ginseng (mean $\pm S E, n=10)$

\begin{tabular}{|c|c|c|c|c|}
\hline 化学性质 Chemical property & CK & A1 & A10 & A20 \\
\hline $\mathrm{pH}$ & $4.69 \pm 0.07^{\mathrm{a}}$ & $4.19 \pm 0.04^{c}$ & $4.49 \pm 0.08^{b}$ & $4.34 \pm 0.04^{\mathrm{bc}}$ \\
\hline 有机质含量 Organic matter content $\left(\mathrm{g} \cdot \mathrm{kg}^{-1}\right)$ & $16.38 \pm 0.91^{\mathrm{a}}$ & $14.43 \pm 0.83^{\mathrm{a}}$ & $13.63 \pm 0.80^{\mathrm{a}}$ & $15.15 \pm 1.02^{\mathrm{a}}$ \\
\hline 全氮含量 Total nitrogen content $\left(\mathrm{g} \cdot \mathrm{kg}^{-1}\right)$ & $0.97 \pm 0.07^{\mathrm{a}}$ & $0.99 \pm 0.07^{\mathrm{a}}$ & $0.89 \pm 0.05^{\mathrm{a}}$ & $1.03 \pm 0.09^{\mathrm{a}}$ \\
\hline 全磷含量 Total phosphorus content $\left(\mathrm{g} \cdot \mathrm{kg}^{-1}\right)$ & $0.84 \pm 0.08^{\mathrm{a}}$ & $0.70 \pm 0.07^{\mathrm{a}}$ & $0.71 \pm 0.05^{\mathrm{a}}$ & $0.92 \pm 0.07^{\mathrm{a}}$ \\
\hline 碱解氮含量 Alkaline hydrolysis nitrogen content $\left(\mathrm{mg} \cdot \mathrm{kg}^{-1}\right)$ & $103.17 \pm 13.79^{\mathrm{a}}$ & $147.84 \pm 21.33^{\mathrm{a}}$ & $131.01 \pm 11.50^{\mathrm{a}}$ & $117.03 \pm 12.67^{\mathrm{a}}$ \\
\hline 有效磷含量 Available phosphorus content $\left(\mathrm{mg} \cdot \mathrm{kg}^{-1}\right)$ & $84.82 \pm 14.10^{\mathrm{a}}$ & $88.72 \pm 24.19^{\mathrm{a}}$ & $94.72 \pm 14.80^{\mathrm{a}}$ & $131.70 \pm 17.49^{\mathrm{a}}$ \\
\hline 有效钾含量 Available potassium content $\left(\mathrm{mg} \cdot \mathrm{kg}^{-1}\right)$ & $123.97 \pm 13.76^{\mathrm{b}}$ & $208.98 \pm 7.65^{\mathrm{a}}$ & $132.97 \pm 13.26^{\mathrm{b}}$ & $142.87 \pm 13.74^{\mathrm{b}}$ \\
\hline
\end{tabular}

$\mathrm{A} 1$, 收获西洋参后轮作 1 年的土壤; $\mathrm{A} 10$, 收获西洋参后轮作 10 年的土壤; $\mathrm{A} 20$, 收获西洋参后轮作 20 年的土壤; $\mathrm{CK}$, 未种植过西洋参的土壤。同一行中 不同小写字母表示样本间差异显著 $(p<0.05)$, 粗体仅表示与CK相比差异显著。

A1, 1-year post-ginseng rotation soil; A10, 10-year post-ginseng rotation soil; A20, 20-year post-ginseng rotation soil; CK, no ginseng cultivation history. Different lowercase letters in the same row indicate significant differences among samples $(p<0.05)$, bold only represents significant difference compared with CK. 


\subsection{2 有机态养分的变化}

经GC-MS检测, 共检出 205 种物质, 其中 24 种 氨基酸类养分含量在各年限土壤间具有显著差异 $(p<0.05)$ 。对比CK, 可以发现收获西洋参后 1 年的 老参地土壤中有 21 种氨基酸类养分的含量下降, 其 中显著下降的有 11 种; 经过长时间的轮作, 鸟氨酸、 丙氨酸、酪氨酸 3 种氨基酸含量得到一定水平的恢复, 而多数氨基酸类养分含量并没有恢复，其中N-乙酰 鸟氨酸、5-氨基戊酸、丝氨酸、亮氨酸、甘氨酸、 组氨酸、焦谷氨酸和苏氨酸 8 种氨基酸在收参 20 年后 老参地中含量依然显著低于对照。同时, 对比CK, 也发现收获西洋参后 1 年的老参地土壤中有 3 种氨基 酸类养分含量上升, 显著增加的有天冬氨酸、正缬 氨酸, 其中正颉氨酸含量经10年轮作后即已恢复到 对照水平, 但天冬氨酸含量始终高于 CK (表3)。

同时, 20 种糖类和糖醇类养分含量在各年限土 壤间具有显著差异 $(p<0.05)$ 。对比CK, 可以发现收 获西洋参后 1 年的老参地土壤中有 19 种糖类和糖醇 类养分的含量下降, 其中显著下降的有 14 种; 经过 长时间的轮作, 其中肌醇半乳糖苷、葡萄糖、双半 乳糖醛酸、麦芽三糖、葡萄糖-1-磷酸、麦芽三醇和 Udp-N-乙酰氨基葡萄糖7种糖类和糖醇类物质含量 得到一定水平的恢复, 而其他糖类和糖醇类养分含 量并没有恢复，其中正丁醇、帕拉金糖醇、松三糖、 甘油和槐糖在收参 20 年后的老参地中含量依然显著 低于对照。同时, 对比CK也发现收获西洋参后 1 年 的老参地土壤中 1,5 -脱水葡萄糖醇含量显著升高, 随轮作时间延长, 其含量逐渐回到对照水平(表3)。 此外, 结果也显示 3 种氨基酸(谷氨酸、 $\mathrm{N}$-氨甲酰天 冬氨酸、 $\beta$-丙氨酸) 和 2 种糖醇类物质(D7葡萄糖、己 六醇)的含量, 在 $\mathrm{A} 1$ 中与 $\mathrm{CK}$ 相比差异不显著, 仅呈 现降低的趋势, 但在A10、A20中却显著低于 $\mathrm{CK}(p<$ 0.05)。

总之, 土壤中部分小分子氨基酸类、糖类和糖 醇类有机态养分含量呈现先降低后逐渐恢复的趋势; 另一部分的含量 20 年轮作后仍继续呈现显著降低的 趋势。

\section{2 不同恢复年限西洋参老参地中酚酸类代谢物 含量}

依据人参属植物的相关研究结果, 本实验选择 了14种具有化感作用潜力的酚酸类代谢物, 进行土 壤提取和HPLC检测, 共检测到10种酚酸类代谢物
(表4), 没食子酸、绿原酸、水杨酸和肉桂酸的含量 低于检出限而未检出。

对上述 10 种酚酸类代谢物进行分析发现，与 $\mathrm{CK}$ 相比，丁香酸和对香豆酸在收获西洋参后 1 年、 10 年和 20 年的老参地中含量均显著下降 $(p<0.05)$, 表明西洋参种植后土壤的丁香酸和对香豆酸含量会 显著降低, 并且经过 10 年和 20 年的轮作恢复, 依然 不能恢复到对照水平。香豆酸在收获西洋参后 1 年、 10 年的老参地土壤中的含量先是显著下降 $(p<0.05)$, 经过 20 年轮作恢复到接近对照水平。原儿茶酸、阿 魏酸和苯甲酸在收获西洋参后 1 年老参地土壤中的 含量显著下降 $(p<0.05)$, 经过 10 年轮作后恢复到接 近对照水平。对羟基苯甲酸、香草酸和香草醛含量 在各组间无显著差异。

\section{3 不同年限土壤中显著变化指标的相关性分析}

Pearson相关性分析显示, 老参地土壤中降低的 多种有机态养分含量、 $\mathrm{pH}$ 和酚酸类代谢物质含量之 间大多数呈显著正相关关系 $(p<0.05)$, 仅有天冬氨 酸含量等较少指标与其他指标间呈现一定的负相关 趋势且相关性不显著 $(p>0.05$, 图2)。

\section{4 不同恢复年限老参地土壤中有机态养分含量 的主成分分析}

氨基酸类和糖醇类有机态养分含量的主成分分 析表明, PC1轴和PC2轴两者共同解释了 $55.0 \%$ 的差 异。刚收获西洋参 1 年的 $\mathrm{A} 1$ 组与 $\mathrm{CK}$ 组的各个样本点 间距较远, 置信椭圆交叉面积最小, 表明 $\mathrm{CK}$ 组与 $\mathrm{A} 1$ 组之间的有机态养分含量存在较为明显的差异; 其他各组老参地与 $\mathrm{CK}$ 相比存在一些差异性, 但也 存在一定的相似性，推测这或许是经过 10 年、 20 年 的轮作土壤中的有机态养分得到部分恢复所造成的 (图3)。

此外, 通过观察图3各个组内样本点的聚集程 度, 发现刚收获西洋参的 $\mathrm{A} 1$ 组各个样本点聚集更加 紧凑, 而其他各组聚集较为松散, 这可能是由于 $3-5$ 年西洋参的种植对于土壤上述有机态养分含量造成 的改变而使得 $\mathrm{A} 1$ 组土壤具有更高的相似性。

\section{3 讨论}

\section{1 不同恢复年限老参地土壤养分变化}

研究结果显示, 与 $\mathrm{CK}$ 相比, 3种老参地土壤 $\mathrm{pH}$ 显著降低, 出现明显酸化, 且经过 20 年轮作依然没 有恢复至对照水平。根据前人研究, 西洋参适宜生 
表3＼cjkstart不同恢复年限老参地土壤小分子有机态养分相对含量的比较(平均值土标准误, $n=10$ )

Table 3 Comparison of relative content of small molecular organic nutrients in soils with different restoration years from the long-term cultivation of American ginseng (mean $\pm S E, n=10)$

\begin{tabular}{|c|c|c|c|c|}
\hline 氨基酸类 Amino acids and amino acid analogues & $\mathrm{CK}$ & $\mathrm{A} 1$ & A10 & $\mathrm{A} 20$ \\
\hline N-乙酰鸟氨酸 N-acetylornithine & $0.24289^{\mathrm{a}}$ & $0.21373^{b}$ & $0.21266^{b}$ & $0.21341^{b}$ \\
\hline 5-氨基戊酸 5-aminovaleric acid & $0.01267^{\mathrm{a}}$ & $0.01124^{\mathrm{b}}$ & $0.01063^{\mathrm{b}}$ & $0.01116^{\mathrm{b}}$ \\
\hline 丝氨酸 Serine & $0.00073^{\mathrm{a}}$ & $0.00051^{b}$ & $0.00055^{\mathrm{b}}$ & $0.00056^{b}$ \\
\hline 亮氨酸 Leucine & $0.00007^{\mathrm{a}}$ & $0.00004^{\mathrm{b}}$ & $0.00004^{\mathrm{b}}$ & $0.00004^{\mathrm{b}}$ \\
\hline 甘氨酸 Glycine & $0.00502^{\mathrm{a}}$ & $0.00284^{c}$ & $0.00535^{\mathrm{a}}$ & $0.00393^{b}$ \\
\hline 组氨酸 Histidine & $0.02444^{\mathrm{a}}$ & $0.00839^{b}$ & $0.01655^{\mathrm{ab}}$ & $0.01167^{b}$ \\
\hline 焦谷氨酸 Pyroglutamic acid & $0.00177^{\mathrm{a}}$ & $0.00046^{b}$ & $0.00177^{\mathrm{a}}$ & $0.00089^{b}$ \\
\hline 苏氨酸 Threonine & $0.00038^{\mathrm{a}}$ & $0.00026^{\mathrm{c}}$ & $0.00036^{\mathrm{ab}}$ & $0.00029^{\mathrm{bc}}$ \\
\hline 鸟氨酸 Ornithine & $0.00634^{\mathrm{a}}$ & $0.00455^{\mathrm{b}}$ & $0.00597^{\mathrm{a}}$ & $0.00558^{\mathrm{a}}$ \\
\hline 丙氨酸 Alanine & $0.01165^{\mathrm{a}}$ & $0.00462^{b}$ & $0.01457^{\mathrm{a}}$ & $0.01009^{\mathrm{ab}}$ \\
\hline 酪氨酸 Tyrosine & $0.00016^{\mathrm{a}}$ & $0.00007^{b}$ & $0.00023^{\mathrm{a}}$ & $0.00016^{\mathrm{a}}$ \\
\hline 谷氨酸 Glutamate & $0.00130^{\mathrm{a}}$ & $0.00124^{\mathrm{a}}$ & $0.00106^{b}$ & $0.00106^{\mathrm{b}}$ \\
\hline $\mathrm{N}$-氨甲酰天冬氨酸 N-carbamoylaspartate & $0.00024^{\mathrm{a}}$ & $0.00022^{\mathrm{ab}}$ & $0.00019^{b}$ & $0.00020^{b}$ \\
\hline$\beta$-丙氨酸 Beta-alanine & $0.00724^{\mathrm{a}}$ & $0.00687^{\mathrm{a}}$ & $0.00583^{b}$ & $0.00575^{b}$ \\
\hline N-甲基丙氨酸 N-methylalanine & $0.01922^{\mathrm{a}}$ & $0.01751^{\mathrm{ab}}$ & $0.01696^{\mathrm{b}}$ & $0.01800^{\mathrm{ab}}$ \\
\hline 氨甲环酸 Tranexamic acid & $0.00083^{\mathrm{a}}$ & $0.00073^{\mathrm{ab}}$ & $0.00074^{\mathrm{ab}}$ & $0.00073^{b}$ \\
\hline 异亮氨酸 Isoleucine & $0.00887^{\mathrm{ab}}$ & $0.00510^{\mathrm{b}}$ & $0.01320^{\mathrm{a}}$ & $0.00899^{\mathrm{ab}}$ \\
\hline L-丝氨酸 L-serine & $0.00220^{\mathrm{ab}}$ & $0.00095^{\mathrm{b}}$ & $0.00339^{\mathrm{a}}$ & $0.00200^{\mathrm{ab}}$ \\
\hline L-苏氨酸 L-threonine & $0.00181^{\mathrm{ab}}$ & $0.00074^{\mathrm{b}}$ & $0.00280^{\mathrm{a}}$ & $0.00168^{\mathrm{ab}}$ \\
\hline 4-氨基丁酸 4-aminobutyric acid & $0.00222^{\mathrm{ab}}$ & $0.00101^{\mathrm{b}}$ & $0.00336^{\mathrm{a}}$ & $0.00272^{\mathrm{ab}}$ \\
\hline 脯氨酸 Proline & $0.00220^{\mathrm{ab}}$ & $0.00092^{\mathrm{b}}$ & $0.00363^{\mathrm{a}}$ & $0.00250^{\mathrm{ab}}$ \\
\hline 天冬酰胺 Asparagine & $0.00246^{\mathrm{a}}$ & $0.00255^{\mathrm{a}}$ & $0.00221^{b}$ & $0.00245^{\mathrm{ab}}$ \\
\hline 天冬氨酸 Aspartate & $0.01107^{\mathrm{b}}$ & $0.01310^{\mathrm{a}}$ & $0.01279^{\mathrm{a}}$ & $0.01375^{\mathrm{a}}$ \\
\hline 正缬氨酸 Norvaline & $0.00201^{\mathrm{b}}$ & $0.00572^{\mathrm{a}}$ & $0.00230^{\mathrm{b}}$ & $0.00302^{\mathrm{b}}$ \\
\hline 甘油 Glycerol & $0.17717^{\mathrm{a}}$ & $0.09779^{b}$ & $0.11746^{b}$ & $0.12596^{b}$ \\
\hline 槐糖 Sophorose & $0.21881^{\mathrm{a}}$ & $0.11203^{b}$ & $0.13339^{b}$ & $0.10229^{b}$ \\
\hline 肌醇半乳糖苷 Galactinol & $0.00507^{\mathrm{a}}$ & $0.00073^{b}$ & $0.00097^{\mathrm{b}}$ & $0.00165^{\mathrm{ab}}$ \\
\hline 正丁醇 1-butanol & $0.00366^{\mathrm{a}}$ & $0.00314^{b}$ & $0.00370^{\mathrm{a}}$ & $0.00333^{b}$ \\
\hline 帕拉金糖醇 Palatinitol & $0.00999^{\mathrm{a}}$ & $0.00741^{b}$ & $0.00959^{\mathrm{ab}}$ & $0.00842^{\mathrm{bc}}$ \\
\hline 松三糖 Melezitose & $0.03672^{\mathrm{a}}$ & $0.01383^{b}$ & $0.02828^{\mathrm{ab}}$ & $0.01930^{\mathrm{bc}}$ \\
\hline 葡萄糖 Glucose & $0.00827^{\mathrm{a}}$ & $0.00369^{b}$ & $0.00758^{\mathrm{a}}$ & $0.00665^{\mathrm{ab}}$ \\
\hline 双半乳糖醛酸 Digalacturonic acid & $0.00684^{\mathrm{a}}$ & $0.00220^{b}$ & $0.00778^{\mathrm{a}}$ & $0.00530^{\mathrm{ab}}$ \\
\hline 麦芽三糖 Maltotriose & $0.03244^{\mathrm{a}}$ & $0.00375^{b}$ & $0.00749^{\mathrm{ab}}$ & $0.00801^{\mathrm{ab}}$ \\
\hline 葡萄糖-1-磷酸 Glucose-1-phosphate & $0.00610^{\mathrm{a}}$ & $0.00289^{b}$ & $0.00598^{\mathrm{a}}$ & $0.00497^{\mathrm{ab}}$ \\
\hline 麦芽三醇 Maltotriitol & $0.00787^{\mathrm{a}}$ & $0.00362^{b}$ & $0.00920^{\mathrm{a}}$ & $0.00587^{\mathrm{ab}}$ \\
\hline Udp-N-乙酰氨基葡萄糖 Udp-N-acetylglucosamine & $0.00606^{\mathrm{a}}$ & $0.00173^{b}$ & $0.00387^{\mathrm{ab}}$ & $0.00378^{\mathrm{ab}}$ \\
\hline D7葡萄糖 D7-glucose & $0.00209^{\mathrm{a}}$ & $0.00201^{\mathrm{a}}$ & $0.00155^{b}$ & $0.00158^{b}$ \\
\hline 己六醇 Hexitol & $0.00240^{\mathrm{a}}$ & $0.00213^{\mathrm{ab}}$ & $0.00195^{\mathrm{b}}$ & $0.00203^{b}$ \\
\hline D-木糖醇 D-xylitol & $0.00485^{\mathrm{ab}}$ & $0.00363^{\mathrm{b}}$ & $0.01268^{\mathrm{a}}$ & $0.00861^{\mathrm{ab}}$ \\
\hline 3-脱氧葡萄糖醇 3-deoxyhexitol & $0.00074^{\mathrm{ab}}$ & $0.00008^{\mathrm{b}}$ & $0.00098^{\mathrm{a}}$ & $0.00046^{\mathrm{ab}}$ \\
\hline 山梨醇 Sorbitol & $0.83454^{\mathrm{ab}}$ & $0.36363^{\mathrm{b}}$ & $1.19804^{\mathrm{a}}$ & $1.23467^{\mathrm{a}}$ \\
\hline 乙二醇 Ethylene glycol & $0.02564^{\mathrm{a}}$ & $0.02176^{\mathrm{ab}}$ & $0.01539^{b}$ & $0.02135^{\mathrm{ab}}$ \\
\hline 甲基 $\beta$-d-吡喃葡萄糖苷 Methyl beta-d-glucopyranoside & $0.04222^{\mathrm{ab}}$ & $0.01158^{\mathrm{b}}$ & $0.06245^{\mathrm{a}}$ & $0.02706^{\mathrm{b}}$ \\
\hline 1,5 -脱水葡萄糖醇 1,5 -anhydroglucitol & $0.00023^{\mathrm{b}}$ & $0.00043^{\mathrm{a}}$ & $0.00025^{\mathrm{b}}$ & $0.00027^{\mathrm{b}}$ \\
\hline
\end{tabular}

$\mathrm{A} 1$, 收获西洋参后轮作 1 年的土壤; $\mathrm{A} 10$, 收获西洋参后轮作 10 年的土壤; $\mathrm{A} 20$, 收获西洋参后轮作 20 年的土壤; $\mathrm{CK}$, 未种植过西洋参的土壤。表中数据 为各代谢物的平均表达量, 无单位, 同一行中不同小写字母表示样本间差异显著 $(p<0.05)$, 粗体仅表示与CK相比差异显著。

A1, 1-year post-ginseng rotation soil; A10, 10-year post-ginseng rotation soil; A20, 20-year post-ginseng rotation soil; CK, no ginseng cultivation history. The data in the table are average expression of each metabolite, unitless, and different lowercase letters in the same row indicate significant differences among samples $(p<0.05)$, bold only represents significant difference compared with CK. 
表4＼cjkstart不同恢复年限老参地土壤酚酸类代谢物含量 $\left(\mathrm{mg} \cdot \mathrm{kg}^{-1}\right.$ )差异比较(平均值土标准误, $\left.n=10\right)$

Table 4 Comparison of phenolic acid metabolites content $\left(\mathrm{mg} \cdot \mathrm{kg}^{-1}\right)$ in soils with different restoration years from the long-term cultivation of American ginseng (mean $\pm S E, n=10)$

\begin{tabular}{|c|c|c|c|c|}
\hline 酚酸类代谢物 Phenolic acid metabolites & CK & A1 & $\mathrm{A} 10$ & $\mathrm{~A} 20$ \\
\hline 香豆酸 Coumalic acid & $0.97 \pm 0.07^{\mathrm{a}}$ & $0.31 \pm 0.04^{c}$ & $0.59 \pm 0.15^{\mathrm{bc}}$ & $0.78 \pm 0.12^{\mathrm{ab}}$ \\
\hline 原儿茶酸 Protocatechuic acid & $0.99 \pm 0.06^{\mathrm{a}}$ & $0.58 \pm 0.08^{b}$ & $0.72 \pm 0.09^{\mathrm{ab}}$ & $0.82 \pm 0.11^{\mathrm{ab}}$ \\
\hline 对羟基苯甲酸 p-Hydroxybenzonic acid & $2.59 \pm 0.07^{\mathrm{a}}$ & $2.17 \pm 0.14^{\mathrm{a}}$ & $2.31 \pm 0.13^{\mathrm{a}}$ & $2.24 \pm 0.18^{\mathrm{a}}$ \\
\hline 香草酸 Vanillic acid & $1.61 \pm 0.09^{\mathrm{a}}$ & $1.46 \pm 0.08^{\mathrm{a}}$ & $1.47 \pm 0.04^{\mathrm{a}}$ & $1.49 \pm 0.10^{\mathrm{a}}$ \\
\hline 咖啡酸 Caffeic acid & $0.02 \pm 0.01^{\mathrm{b}}$ & $0.23 \pm 0.15^{\mathrm{b}}$ & $0.01 \pm 0.01^{\mathrm{b}}$ & $0.74 \pm 0.22^{\mathrm{a}}$ \\
\hline 丁香酸 Syringic acid & $2.33 \pm 0.14^{\mathrm{a}}$ & $1.55 \pm 0.13^{b}$ & $1.66 \pm 0.14^{b}$ & $1.48 \pm 0.17^{b}$ \\
\hline 香草醛 Vanillin & $0.87 \pm 0.06^{\mathrm{a}}$ & $1.00 \pm 0.12^{\mathrm{a}}$ & $0.89 \pm 0.06^{\mathrm{a}}$ & $0.87 \pm 0.07^{\mathrm{a}}$ \\
\hline 对香豆酸 $p$-Coumaric acid & $3.52 \pm 0.22^{\mathrm{a}}$ & $2.06 \pm 0.35^{b}$ & $2.43 \pm 0.15^{b}$ & $2.71 \pm 0.21^{b}$ \\
\hline 阿魏酸 Ferulic acid & $1.51 \pm 0.12^{\mathrm{a}}$ & $0.85 \pm 0.16^{b}$ & $1.19 \pm 0.08^{\mathrm{ab}}$ & $1.34 \pm 0.09^{\mathrm{ab}}$ \\
\hline 苯甲酸 Benzoic acid & $2.35 \pm 0.37^{\mathrm{a}}$ & $1.29 \pm 0.37^{\mathrm{b}}$ & $2.28 \pm 0.27^{\mathrm{a}}$ & $2.91 \pm 0.34^{\mathrm{a}}$ \\
\hline
\end{tabular}

$\mathrm{A} 1$, 收获西洋参后轮作 1 年的土壤; $\mathrm{A} 10$, 收获西洋参后轮作 10 年的土壤; $\mathrm{A} 20$, 收获西洋参后轮作 20 年的土壤; $\mathrm{CK}$, 未种植过西洋参的土壤。同一行中 不同小写字母表示样本间差异显著 $(p<0.05)$, 粗体仅表示与CK相比差异显著。

A1, 1-year post-ginseng rotation soil; A10, 10-year post-ginseng rotation soil; A20, 20-year post-ginseng rotation soil; CK, no ginseng cultivation history. Different lowercase letters in the same row indicate significant differences among samples $(p<0.05)$, bold only represents significant difference compared with CK.

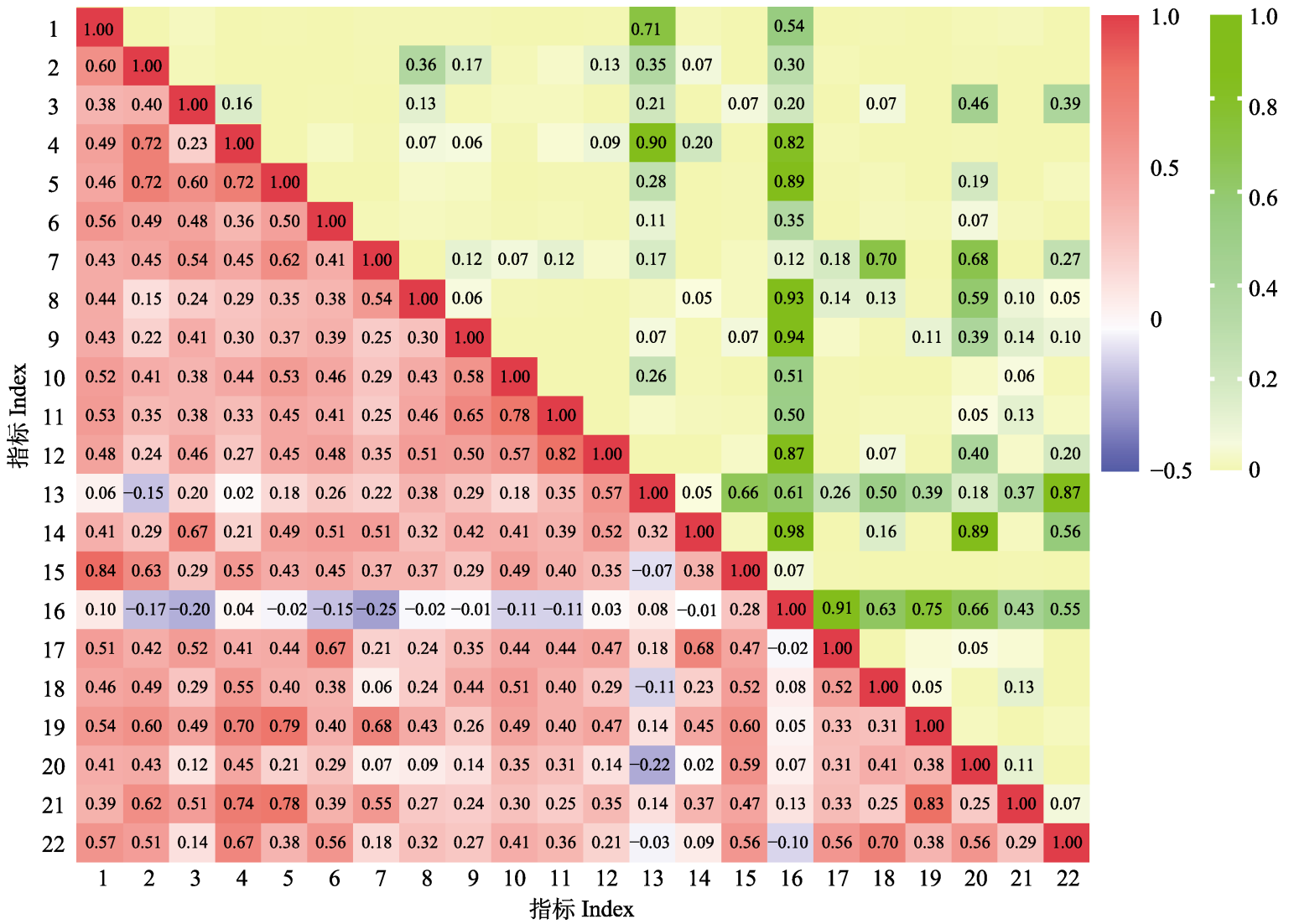

图2 不同恢复年限老参地土壤中显著变化指标的Pearson相关性热图。左下方为Pearson相关性热图, 格子内数字表示相关性 $(r)$; 右上方为相关显著性热图, 将其中 $p>0.05$ 的数值在格子中标出, 表示两种指标的Pearson相关不显著。1, 甘油; 2 , 槐糖; 3 , 正丁醇; 4 , 麦芽三醇; 5 , 帕拉金糖醇; 6 , 松三糖; $7, \mathrm{pH} ; 8$, 香豆酸; 9 , 原儿茶酸; 10 , 丁香酸; 11 , 对香豆酸; 12 , 阿魏酸; 13 , 苯 甲酸; 14 , 甘氨酸; $15, \mathrm{~N}$-乙酰鸟氨酸; 16 , 天冬氨酸; 17 , 苏氨酸; 18 , 丝氨酸; 19 , 组氨酸; 20 , 谷氨酸; 21 , 焦谷氨酸; 22 , 亮氨酸。 Fig. 2 Pearson correlation heat map of significant changed indices in soils with different restoration years from the long-term cultivation of American ginseng. The lower left is the Pearson correlation heat map, and the number in the grid represents the correlation coefficient $(r)$; the top right is the correlation significance heat map, in which the value of $p>0.05$ is marked, indicating that the Pearson correlation of the two indicators is non-significant. 1, glycerol; 2, sophorose; 3, 1-butanol; 4, maltotriitol; 5, palatinitol; 6, melezitose; 7, pH; 8, coumaric acid; 9, protocatechuic acid; 10, syringic acid; 11, p-coumaric acid; 12, ferulic acid; 13, benzoic acid; 14, glycine; 15, N-acetylornithine; 16, aspartate; 17, threonine; 18, serine; 19, histidine; 20, glutamate; 21, pyroglutamic acid; 22, leucine. 


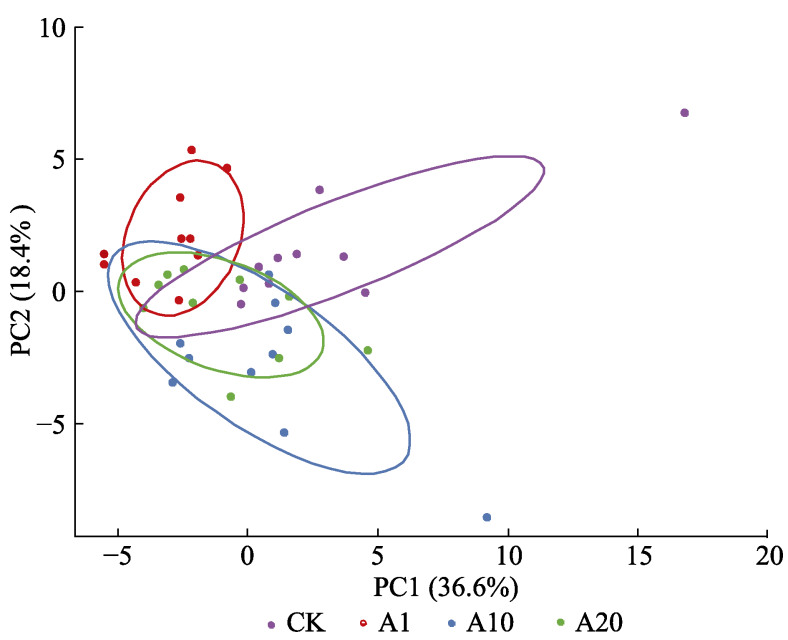

图3 不同恢复年限老参地土壤氨基酸类和糖醇类养分含量 的主成分分析 $(\mathrm{PCA})$ 。 $\mathrm{A} 1$, 收获西洋参后轮作 1 年的土壤; $\mathrm{A} 10$, 收获西洋参后轮作 10 年的土壤; A20, 收获西洋参后轮 作 20 年的土壤; CK, 未种植过西洋参的土壤。两点之间的距 离越短, 表明两组养分含量组成的相似性越高; 反之, 两点 之间的距离越远, 两组养分含量的差异性越大; 置信椭圆也 具有相同的含义。

Fig. 3 Principal component analysis (PCA) of soil amino acids and sugar alcohols contents in soils with different restoration years from the long-term cultivation of American ginseng. A1, 1-year post-ginseng rotation soil; A10, 10-year post-ginseng rotation soil; A20, 20-year post-ginseng rotation soil; CK, no ginseng cultivation history. The shorter the distance between the two points, the higher the similarity of nutrient content between the two groups; on the contrary, the farther the distance between the two points, the greater the difference of nutrient content between the two groups; the confidence ellipse has the same meaning.

长的 $\mathrm{pH}$ 范围为中性至弱酸性(徐厚来, 2014), 土壤 酸化不利于西洋参的生长(Rahman \& Punja, 2005)。 山东省威海市文登区土壤偏酸性, 栽种一茬西洋参 后土壤酸化加重变得不适合西洋参的生长, 经过 20 年的轮作后 $\mathrm{pH}$ 依然显著低于对照, 表明西洋参对于 土壤 $\mathrm{pH}$ 的影响具有一定的持久性, 土壤酸化及其关 联效应可能是造成西洋参长期连作障碍的一个关键 限制因子。土壤有效钾含量在不同种类间具有显著 的差异, 其中, 收获西洋参后 1 年的老参地土壤(A1) 的有效钾含量显著高于其余 3 组, 推测是因为 $3-4$ 龄 西洋参对于钾肥需求较高(蔡荟梅等, 2007; 孙贺, 2008), 种植者对土壤进行钾肥追加所造成的增高 的现象。除此之外, 土壤基本化学性质在各年限土 壤间没有明显差异, 表明土壤无机态养分虽然是影 响植物生长的重要因素, 但西洋参连作障碍并非是 由无机态养分贵缺而引起的。

植物对于土壤养分的摄取不仅只有无机态养分, 也包括溶解性有机氮, 如氨基酸态氮(Fenn et al.,
1998; Chapin III et al., 2011)、溶解性小分子糖类等 (Kuzyakov \& Jones, 2006)等有机态养分。本研究结 果显示, 收获西洋参后 1 年的老参地(A1)土壤中的 12 种氨基酸类物质、 12 种糖类和糖醇类物质的含量 显著下降, 其中 8 种氨基酸、 5 种糖类和糖醇类物质 含量在A1中下降后, 在A20中依然没有恢复至 CK 水平。丁双双等(2016)的研究发现, 叶面喷施糖醇 (甘露醇、木糖醇)和氨基酸(甘氨酸、谷氨酸)具有增 加芸苔(Brassica rapa var. oleifera) 的生物量、改善其 品质和促进其养分吸收的作用。多位研究者进行养 分施加实验，也发现有机态养分对于受试植物具有 不同程度的促生长作用(刘赵帆等, 2013; 于会丽等, 2014; 杜伟等, 2015)。据Shen等(2019)关于高山植物 对 $\mathrm{N}$ 沉降 (大气中的 $\mathrm{N}$ 转化为 $\mathrm{NH}_{\mathrm{x}}$ 和 $\mathrm{NO}_{\mathrm{x}}$ 的形式进入 土壤和水体)响应的研究发现, 更低的 $\mathrm{N}$ 沉降对草本 植物的光合作用和生长具有不利影响; 且Guo等 (2020)也发现了具有较长非生长期的植物, 如高山 植物以及苔原植物对于土壤中的有机态 $\mathrm{N}$ (氨基酸) 具有更大的偏好, 并且小分子糖类和糖醇类同样能 被植物摄取。对于在较高纬度生长的宿根植物西洋 参而言, 氨基酸类、糖类和糖醇类可以为其秋末到 春初长达近 6 个月的非生长期提供一定的养分支持, 因此, 推测氨基酸、小分子糖和糖醇类物质的下降 会在养分上限制药用植物西洋参的重茬生长, 且部 分氨基酸类、糖类和糖醇类物质经过 20 年轮作依然 难以恢复, 这可能是西洋参连作障碍可持续数十年 的重要原因之一。

\section{2 不同恢复年限老参地土壤中酚酸类代谢物含量 的变化}

已有对具有连作障碍的植物(如茄科、蓄薇科等) 的研究发现, 土壤中酚酸类代谢物具有化感自毒作 用, 酚酸类代谢物随连作时间延长逐渐积累到达活 性浓度, 进而造成自毒作用威胁该种植物的重茬种 植(甄文超等, 2004; 李雪枫等, 2019)。有研究者对西 洋参进行一定浓度的生物测定实验也发现了酚酸类 代谢物的化感自毒作用(高微微等, 2006a; 焦晓林 等, 2015a)。然而本实验结果显示, 6种酚酸类代谢物 香豆酸、原儿茶酸、丁香酸、对香豆酸、阿魏酸和 苯甲酸的含量在收获西洋参后 1 年的老参地土壤中 均显著降低, 其中香豆酸、原儿茶酸、阿魏酸和苯 甲酸的含量随着轮作时间延长逐渐恢复到对照水平, 但丁香酸、对香豆酸的含量即使经过 20 年的轮作后 
依然没有恢复到对照水平。根据已有研究发现, 香 豆酸、肉桂酸等多种酚酸可以在低浓度下促进西洋 参致病菌Rhizoctonia solani 、Fusarium solani 和 Cylindrocarpon destructans的生长; 但在高浓度下对 其有抑制作用(Yang \& Gao, 2009; 李自博等, 2016); Yang和Gao (2009)也发现, 多种酚酸类代谢物在高 浓度下能显著抑制Phytophthora cactorum (一种引 起西洋参疫霉病的病原菌)的增殖, 其中对香豆酸 强烈抑制了真菌的体外生长, 显著减少了链格孢霉 Alternaria alternata引起的腐烂(Yuan et al., 2019)。 可见, 酚酸类代谢物在较高浓度下对病原菌有抑制 作用, 在低浓度下则可能有促进作用。因此, 我们认 为, 土壤中保持较高浓度的酚酸可能会通过抑制有 害菌生长而间接地保护西洋参, 即较高浓度的酚酸 对西洋参生长有利。

在作物连作障碍的研究中, 酚酸含量下降的现 象也并非首次被发现, 如何志贵(2019)以及孙文庆 (2017)等分别发现种植一茬半夏(Pinellia ternata)、 番茄(Lycopersicon esculentum)后土壤中酚酸含量显 著下降。而Jiao等(2012)在土壤中添加西洋参根残体 后也发现了类似的现象。我们的研究在一定程度上 支持了上述推论。生产实践中, 种植者会在春季西 洋参发芽前用麦秸覆盖土壤, 除保持土壤温度和水 分外, 还有研究发现麦秸覆盖会增加土壤中酚酸类 代谢物的浓度(郑皓皓, 2001; Jia et al., 2004)。我们 的研究为这一种植管理措施提供了一定的科学解释。

\section{4 结论}

本研究比较了不同年限老参地土壤养分以及酚 酸类代谢物含量的差异, 发现3种类型老参地土壤 酸化严重, 土壤中氨基酸类、糖类和糖醇类有机态 养分以及酚酸类代谢物含量均下降, 且部分有机态 养分和酚酸类代谢物含量在下降后具有一定的持久 性, 难以通过轮作恢复。结合Pearson相关性分析, 可以发现老参地土壤中多种有机态养分含量以及 酚酸类代谢物含量降低, 并且有机态养分含量与 $\mathrm{pH}$ 、酚酸类代谢物含量之间呈显著正相关关系, 即 各土壤指标之间存在密切的交互关系。综上所述, 种植西洋参引起的长期土壤酸化、有机态养分缺失 和具有抑菌作用的酚酸含量的降低及各因子间的 协同作用可能是西洋参连作障碍的关键因素, 这 在一定程度上为西洋参连作障碍可持续数十年提
供了一定解释。

致谢 感谢威海文登传福参业有限公司对实验样品 采集的大力支持! 感谢鲁东大学金成武老师、张建 龙老师对液相实验的指导和帮助!

\section{参考文献}

Bao SD (2000). Soil Agrochemical Analysis. 3rd ed. China Agriculture Press, Beijing. 25-108. [鲍士旦 (2000), 土壤 农化分析. 3版. 中国农业出版社, 北京. 25-108.]

Bi W (2008). Identification of the Pathogens Causing the Root Rot and Their Pathogenicity on American Ginseng in Beijing. Master degree dissertation, Peking Union Medical College, Beijing. 32-56. [毕武 (2008). 北京地区西洋参 根腐病相关真菌的研究. 硕士学位论文, 北京协和医学 院, 北京. 32-56.]

Bi XB, Yang JX, Gao WW (2010). Autotoxicity of phenolic compounds from the soil of American ginseng (Panax quinquefolium L.). Allelopathy Journal, 25, 115-121.

Cai HM, Zang AX, Xia C, Cai JM (2007). Study on the relationships between Panax quinquefolius L. output and soil fertility, microorganism and enzyme activity. Journal of Anhui Agricultural Sciences, 26, 8267-8268. [蔡荟梅, 藏 爱香, 夏春, 蔡敬民 (2007). 西洋参产量与土壤肥 力·微生物和酶活性关系的研究. 安徽农业科学, 26 , 8267-8268.]

Chapin III FS, Matson PA, Mooney HA (2011). Principles of Terrestrial Ecosystem Ecology. 2nd ed. Springer, Berlin. 586-623.

Chen CF, Chiou WF, Zhang JT (2008). Comparison of the pharmacological effects of Panax ginseng and Panax quinquefolium. Acta Pharmacologica Sinica, 29, 1103-1108.

Chen SL, Zhou YQ, Xie CX, Zhao RH, Sun CZ, Wei JH, Liu ZQ, Gao WW (2008). Suitability evaluation of Panax quinquefolium's producing area based on TCMGIS-I. China Journal of Chinese Materia Medica, 33, 741-745. [陈士林, 周应群, 谢彩香, 赵润怀, 孙成忠, 魏建和, 刘召芹, 高微微 (2008). 基于TCMGIS-I的西洋参生态 适宜性分析. 中国中药杂志, 33, 741-745.]

Ding SS, Li YT, Yuan L, Zhao BQ, Lin ZA, Yang XD, Li J, Zhang JJ (2016). Effects of sugar alcohols and amino acids on growth, quality and calcium nutrition of Chinese cabbage. Journal of Plant Nutrition and Fertilizer, 22, 744-751. [丁双双, 李燕婷, 袁亮, 赵秉强, 林治安, 杨 相东, 李娟, 张建君 (2016). 糖醇和氨基酸对小白菜钘 营养及生长、品质的影响. 植物营养与肥料学报, 22 , 744-751.]

Dong LL, Xu J, Zhang LJ, Yang J, Liao BS, Li XW, Chen SL (2017). High-throughput sequencing technology reveals that continuous cropping of American ginseng results in changes in the microbial community in arable soil.

www.plant-ecology.com 
Chinese Medicine, 12, 1-11.

Du W, Zhao BQ, Lin ZA, Yuan L, Li YT (2015). Study on the enhancement and mechanism of organic-inorganic compound fertilizer on inorganic fertilizer utilization III. Effect of potassium sulfate combined with organic material on maize yield and $\mathrm{K}$ fertilizer utilization. Journal of Plant Nutrition and Fertilizer, 21, 58-63. [杜伟, 赵秉强, 林治 安, 袁亮, 李燕婷 (2015). 有机无机复混肥优化化肥养 分利用的效应与机理研究III. 有机物料与钾肥复混对 玉米产量及肥料养分吸收利用的影响. 植物营养与肥 料学报, 21, 58-63.]

Farh MEA, Kim YJ, Yang DC (2018). Cylindrocarpon destructans/Ilyonectria radicicola-species complex: causative agent of ginseng root-rot disease and rusty symptoms. Journal of Ginseng Research, 42, 9-15.

Fenn ME, Poth MA, Aber JD, Baron JS, Bormann BT, Johnson DW, Lemly AD, McNulty SG, Ryan DF, Stottlemyer R (1998). Nitrogen excess in North American ecosystems: predisposing factors, ecosystem responses, and management strategies. Ecological Applications, 8, 706-733.

Gao WW, Tong JM, Guo SX (2006a). Research progress on ecological functions of plant secondary metabolites. Chinese Pharmaceutical Journal, 41, 961-964. [高微微, 佟建 明, 郭顺星 (2006a). 植物次生代谢产物的生态学功能 研究进展. 中国药学杂志, 41, 961-964.]

Gao WW, Zhao YJ, Wang YP, Chen SL (2006b). A review of research on sustainable utilization of medicinal plant cultivated land in China. China Journal of Chinese Materia Medica, 31, 1665-1669. [高微微, 赵杨景, 王玉萍, 陈士 林 (2006b). 我国药用植物栽培地的可持续利用研究. 中国中药杂志, 31, 1665-1669.]

Gao ZQ, Zhang SX (1998). Continuous cropping obstacle and rhizospheric microecology I. Root exudates and their ecological effects. Chinese Journal of Applied Ecology, 9, 3-5. [高子勤, 张淑香 (1998). 连作障碍与根际微生态 研究I. 根系分泌物及其生态效应. 应用生态学报, 9, 3-5.]

Guo WJ, Zhang ZL, Liu Q, Xiao J, Yin HJ (2020). Seasonal variations in plant nitrogen acquisition in an ectomycorrhizal alpine forest on the eastern Tibetan Plateau, China. Plant and Soil, 459, 79-91.

Hartley RD, Buchan H (1979). High-performance liquid chromatography of phenolic acids and aldehydes derived from plants or from the decomposition of organic matter in soil. Journal of Chromatography A, 180, 139-143.

He CN, Gao WW, Yang JX, Bi W, Zhang XS, Zhao YJ (2009). Identification of autotoxic compounds from fibrous roots of Panax quinquefolium L. Plant and Soil, 318, 63-72.

He ZG (2019). Mechanism of Continuous Cropping Obstacles and Crop Rotation Restoration in Pinellia ternate. $\mathrm{PhD}$ dissertation, Northwest A\&F University, Yangling, Shaanxi. 38-49. [何志贵 (2019). 半夏连作障碍发生机
制与轮作修复研究. 博士学位论文, 西北农林科技大 学, 陕西杨凌. 38-49.]

Jia CH, Wang P, Zhao XQ (2004). Change of phenolic acids concentration in soil under wheat straw mulch and the effect of phenolic acids on early growth stage of summer maize. Acta Agriculturae Boreali-Sinica, 4, 84-87.

Jian ZY, Wang WQ, Meng L, Zhang ZL (2008). Research progress on continuous cropping obstacles of Panax medicinal plants. Modem Chinese Medicine, 10, 3-5. [简在友, 王文全, 孟丽, 张子龙 (2008). 人参属药用植物连作障 碍研究进展. 中国现代中药, 10, 3-5.]

Jiao XL, Bi XB, Gao WW (2015a). Allelopathic effect of p-coumaric acid on American ginseng and its physiological mechanism. Acta Ecologica Sinica, 35, 3006-3013. [焦 晓林, 毕晓宝, 高微微 (2015a). p-香豆酸对西洋参的化 感作用及生理机制. 生态学报, 35, 3006-3013.]

Jiao XL, Du J, Bi XB, Gao WW (2015b). Effect of phenolic acid and ginsenosides in American ginseng on the growth of pathogenic fungi. Chinese Agricultural Science Bulletin, 31，105-110. [焦晓林, 杜静, 毕晓宝, 高微微 (2015b). 西洋参中酚酸及㿝苷成分对病原菌的作用. 中 国农学通报, 31, 105-110.]

Jiao XL, Du J, Gao WW (2012). Autotoxicity and promoting: dual effects of root litter on American ginseng growth. Acta Ecologica Sinica, 32, 3128-3135.

Kuzyakov Y, Jones DL (2006). Glucose uptake by maize roots and its transformation in the rhizosphere. Soil Biology \& Biochemistry, 38, 851-860.

Lei FJ, Zhang AH, Zhang QJ, Zhang LX (2010). Advances in research on allelopathy of ginseng and American ginseng. China Journal of Chinese Materia Medica, 35, 2221-2226. [雷锋杰, 张爱华, 张秋菊, 张连学 (2010). 人参、西洋 参化感作用研究进展. 中国中药杂志, 35, 2221-2226.]

Li CW, Chen GX, Zhang JL, Zhu P, Bai XF, Hou YP, Zhang XX (2021). The comprehensive changes in soil properties are continuous cropping obstacles associated with American ginseng (Panax quinquefolius) cultivation. Scientific Reports, 11, 5068. DOI: 10.1038/s41598-021-84436-x.

Li JF, Shao HH, Bi YM, Jiao XL, Zhang XM, Gao WW (2020). Effects of nutrients deficiency on growth and saponin accumulation of American ginseng. China Journal of Chinese Materia Medica, 45, 1866-1872.

Li L, Jiang JL (2018). Research advances in allelopathic autotoxicity and continuous cropping obstacle of American ginseng. Molecular Plant Breeding, 16, 4436-4443. [李丽, 蒋景龙 (2018). 西洋参化感自毒作用与连作障碍研究 进展. 分子植物育种, 16, 4436-4443.]

Li XF, Wang J, Hu J, Ye XY, Zhang XT (2019). Research progress on allelopathy of melon vegetables. North Horticulture, 16, 136-145. [李雪枫, 王坚, 胡坚, 叶晓园, 张秀 婷 (2019). 瓜类蔬菜化感作用研究进展. 北方园艺，16, 136-145.] 
Li ZB, Zhou RJ, Xie YJ, Fu JF (2016). Allelopathic effects of phenolic compounds of ginseng root rhizosphere on Cylindrocarpon destructans. Chinese Journal of Applied Ecology, 27，3616-3622. [李自博, 周如军, 解宇娇, 傅 俊范 (2016). 人参连作根际土壤中酚酸物质对人参锈 腐病菌的化感效应. 应用生态学报, 27, 3616-3622.]

Liu ZF, Zhang GB, Yu JH, Yang HX, Shi GY, Ma YX, Li J (2013). Effects of different nitrogen forms and their ratios on broccoli yield, quality, and nutrient absorption. Chinese Journal of Applied Ecology, 24, 1923-1930. [刘赵帆, 张 国斌，郁继华，杨海兴，师桂英，马彦霞，李杰 (2013). 氮肥形态及配比对花椰菜产量、品质和养分吸收的影 响. 应用生态学报, 24, 1923-1930.]

Lu RK (2000). Soil Agricultural Chemical Analysis Method. China Agricultural Science and Technology Press, Beijing. 23-145. [鲁如坤 (2000). 土壤农业化学分析方法. 中国 农业科技出版社，北京. 23-145.]

Ma BL, Qin XH, Shi ZX, Liu CY (2011). The research progress of American ginseng clinical pharmacology. Journal of Jiangxi University of Traditional Chinese Medicine, 23, 88-29. [马宝兰, 秦绪花, 史载祥, 刘春援 (2011). 西洋 参临床药理研究进展(2004-2010). 江西中医学院学报, 23, 88-92.]

Momoshima N, Tjahaja PI, Takashima Y (1991). Root uptake of ${ }^{14} \mathrm{C}$-labeled glucose by radish seedling. Radioisotopes, 40, 64-66.

Rahman M, Punja ZK (2005). Factors influencing development of root rot on ginseng caused by Cylindrocarpon destructans. Phytopathology, 95, 1381-1390.

Shen H, Dong SK, Li S, Xiao JN, Han YH, Yang MY, Zhang J, Gao XX, Xu YD, Li Y, Zhi YL, Liu SL, Dong QM, Zhou HK, Yeomans JC (2019). Effects of simulated N deposition on photosynthesis and productivity of key plants from different functional groups of alpine meadow on QinghaiTibetan Plateau. Environmental Pollution, 251, 731-737.

Sun H (2008). The Research of Panax quinquefolium Linne's Nutrient Accumulation Rule. Master degree dissertation, Jilin Agricultural University, Changchun. [孙贺 (2008). 西洋参养分积累规律的研究. 硕士学位论文, 吉林农业 大学, 长春.]

Sun WQ (2017). Identification of Phenolic Acids in Processing Tomato Andeffects on Growth of Phenolic Acids. Master degree dissertation, Shihezi University, Shihezi, Xinjiang. 8-30. [孙文庆 (2017). 加工番茄酚酸类化感物质的鉴定 及对幼苗生长的影响. 硕士学位论文, 石河子大学, 新 疆石河子. 8-30.]

Vitousek PM, Matson PA (1988). Nitrogen transformations in a range of tropical forest soils. Soil Biology \& Biochemistry, 20, 361-367.

Xu HL (2014). Preliminary study on soil $\mathrm{pH}$ and continuous cropping obstacles. Chinese Medicine Modern Distance Education of China, 12(3), 148. [徐厚来 (2014). 参地土
壤 $\mathrm{pH}$ 值与连作障碍问题初探. 中国中医药现代远程教 育, 12(3), 148.]

Yang JX (2009). Allelopathy of Phenolic Acids from Panax quinquefolium and its Influencing Factors. Master degree dissertation, China Union Medical College, Beijing. [杨家 学 (2009). 西洋参酚酸类化合物的化感作用及影响因 子研究. 硕士学位论文, 中国协和医科大学, 北京.]

Yang JX, Gao WW (2009). Effects of phenolic allelochemicals on the pathogen of Panax quiquefolium L. Chinese Agricultural Science Bulletin, 25, 207-211.

Yu HL, Lin ZA, Li YT, Yuan L, Zhao BQ (2014). Effects of spraying low molecular organic compounds on growth and nutrients uptake of rape (Brassica chinensis L.). Journal of Plant Nutrition and Fertilizer, 20, 1560-1568. [于会丽, 林治安, 李燕婷, 袁亮, 赵秉强 (2014). 喷施小分子有 机物对小油菜生长发育和养分吸收的影响. 植物营养 与肥料学报, 20, 1560-1568.]

Yuan SZ, Li WS, Li QQ, Wang LM, Cao JK, Jiang WB (2019). Defense responses, induced by $p$-coumaric acid and methyl $p$-coumarate, of jujube (Ziziphus jujuba Mill.) fruit against black spot rot caused by Alternaria alternata. Journal of Agricultural and Food Chemistry, 67, 2801-2810.

Zhang FS, Cao YP (1992). Rhizosphere dynamics and plant nutrition. Acta Pedologica Sinica, 29, 239-250. [张福锁, 曹一平 (1992). 根际动态过程与植物营养. 土壤学报, 29, 239-250.]

Zhang GZ, Zhang SF (2004). Pathogenicity of fungi isolated from American ginseng seeds and bioassay of fungicides against the pathogenic fungi. China Journal of Chinese Materia Medica, (2), 47-50. [张国珍, 张树峰 (2004). 西 洋参种子分离真菌的致病性测定及杀菌剂对种传病原 真菌的生物测定. 中国中药杂志, (2), 47-50.]

Zhang XS (2013). Abatement Effect of Crop Rotation on Replant Problem of Panax quinquefolium L. PhD dissertation, Peking Union Medical College, Beijing. [张雪松 (2013). 轮作对西洋参连作障碍消减作用的研究. 博士 学位论文, 北京协和医学院, 北京.]

Zhen WC, Wang XY, Kong JY, Cao KQ (2004). Determination of phenolic acids in root exudates and decomposing products of strawberry and their allelopathy. Journal of Agricultural University of Hebei, 27, 74-78. [甄文超, 王晓燕, 孔俊 英, 曹克强 (2004). 草莓根系分泌物和腐解物中的酚酸 类物质及其化感作用. 河北农业大学学报, 27, 74-78.]

Zheng HH, Hu XJ, Jia JY, Wu E, Xing JJ (2001). Changes in phenolic acid in plough layer and its effects on the growth and yield of summer corn with returning wheat straw. Chinese Journal of Eco-Agriculture, (4), 83-85. [郑皓皓, 胡晓军, 贾敬业, 吴荌, 邢建军 (2001). 麦秸还田耕层 酚酸变化及其对夏玉米生长的影响. 中国生态农业学 报, (4), 83-85.]

责任编委: 程晓莉 责任编辑: 李 敏

www.plant-ecology.com 